\title{
Immunotherapy - 2066. A study on skin prick test patterns of atopic patients managed in a tertiary care setting of Sri Lanka
}

\author{
Anoma Siribaddana ${ }^{1 *}$, Fariha Sitheeque ${ }^{2}$, Sonali Surangi ${ }^{3}$, Srinath Illeperuma ${ }^{1}$, Dinesh Dassanayaka ${ }^{1}$ \\ From 2nd WAO International Scientific Conference (WISC 2012) \\ Hyderabad, India. 6-9 December 2012
}

\section{Background}

Studies of common allergens causing allergic rhinitis and other atopic conditions in Sri Lanka are scarce. We used skin prick testing to study the pattern of allergic sensitization in Sri Lankan patients having allergic rhinitis.

\section{Methods}

We performed skin prick test (SPT) using 19 allergens on 54 patients having allergic rhinitis (AR). AR was classified according to AREA classification ${ }^{1}$. Presence of coexisting Asthma and allergic conjunctivitis was documented. The severity of asthma was assessed using the GINA guidelines. Informed written consent was obtained by a member of the research team. SPT was performed using a panel of 19 allergens. Positive control was done using a standard solution of histamine and the negative was performed with glycerol buffer. The resultant induration was measured using a standard scale and recorded in millimeters. A wheel of $3 \mathrm{~mm}$ was considered as positive. The results were analyzed in percentages.

\section{Results}

We studied 25 males and 29 females. The age range of patients was from 09 to 68 yrs with a mean age of 33.5yrs. A positive reaction to at least one allergen was seen in $87.1 \%$ of patients. Intermittent Rhinitis was seen in $53.7 \%$ and persistent disease was seen in $46.3 \%$. Moderate to severe disease was found in $72.2 \%$ and the rest had mild disease. Coexisting asthma was seen in $43.6 \%$ and conjunctivitis was seen in $29.6 \%$ of the study population. The results of SPT expressed in percentages showed, Dermatophagoides farina spp. 59.3\%, Dermatophagoides pteronyssinus spp. 63\%, Wheat Dust 33\%, Cotton Gin 33\%, Rice Dust 14\%, Aspergillus flavus 18.5\%, Aspergillus fumigates 7.4\% Aspergillus niger 11.1\%, Aspergillus glaucus/amstelodami $11 \%$, Tricho. mentagrophytes $7.4 \%$, Candida spp. 18.5\%, Cladosporum herbarium 11\%, Epidermo floccosum 22.2\%, German Cockroach 51.9\%, American Cockroach 40.7\%, Bermuda Grass 11\%, Red Mulberry 7.4\%, White Mulberry $11.1 \%$ and Acacia 3.7\% positivity.

\section{Conclusions}

The commonest allergen sensitizing this cohort of patients was of the Dermatophagoides pteronyssinus, followed by the Dermatophagoides farina. Next common were the German Cockroach 51.9\% and American Cockroach 40.7\%. The least common was of Acacia.

\section{Author details}

${ }^{1}$ Respiratory Medicine, Teaching Hospital Kandy, Kandy, Sri Lanka. ${ }^{2}$ Teaching Hospital Kandy, Sri Lanka. ${ }^{3}$ Department of Physiology, Faculty of Medicine, Peradeniya, Sri Lanka.

Published: 23 April 2013

\section{doi:10.1186/1939-4551-6-S1-P149}

Cite this article as: Siribaddana et al:: Immunotherapy - 2066. A study on skin prick test patterns of atopic patients managed in a tertiary care setting of Sri Lanka. World Allergy Organization Journal 2013 6(Suppl 1): P149.

${ }^{1}$ Respiratory Medicine, Teaching Hospital Kandy, Kandy, Sri Lanka

Full list of author information is available at the end of the article

(c) 2013 Siribaddana et al; licensee BioMed Central Ltd. This is an Open Access article distributed under the terms of the Creative 